\title{
Abundances of the light elements from UV (HST) and red (ESO) spectra in the very old star HD $84937^{\star}$
}

\author{
M. Spite ${ }^{1}$, R. C. Peterson ${ }^{2}$, A. J. Gallagher ${ }^{1}$, B. Barbuy ${ }^{3}$, and F. Spite ${ }^{1}$ \\ 1 GEPI, Observatoire de Paris, PSL Research University, CNRS, Université Paris Diderot, Sorbonne Paris Cité, Place Jules Janssen, \\ 92195 Meudon, France \\ e-mail: monique.spite@obspm. fr \\ 2 SETI Institute, 189 Bernardo Ave, Suite 100, Mountain View, CA 94043, USA \\ 3 Universidade de São Paulo, IAG, Rua do Matão 1226, Cidade Universitária, 05508-900 São Paulo, Brazil
}

Received 14 November 2016 / Accepted 5 January 2017

\begin{abstract}
Aims. In order to provide a better basis for the study of mechanisms of nucleosynthesis of the light elements beyond hydrogen and helium in the oldest stars, the abundances of $\mathrm{C}, \mathrm{O}, \mathrm{Mg}, \mathrm{Si}, \mathrm{P}, \mathrm{S}, \mathrm{K}$, and $\mathrm{Ca}$ have been derived from UV-HST and visible-ESO high resolution spectra in the old, very metal-poor star HD 84937, at a metallicity that is $1 / 200$ that of the Sun's. For this halo main-sequence turnoff star, the abundance determination of $\mathrm{P}$ and $\mathrm{S}$ are the first published determinations.

Methods. The LTE profiles of the lines were computed and fitted to the observed spectra. Wherever possible, we compared the abundances derived from the UV spectrum to abundances derived from the visible or near-infrared spectra, and also corrected the derived abundances for non-LTE effects. Three-dimensional (3D) $\mathrm{CO}^{5} \mathrm{BOLD}$ model atmospheres have been used to determine the abundances of $\mathrm{C}$ and $\mathrm{O}$ from molecular $\mathrm{CH}$ and $\mathrm{OH}$ bands.

Results. The abundances of these light elements relative to iron in HD 84937 are found to agree well with the abundances of these elements in classical metal-poor stars. Our HD 84937 carbon abundance determination points toward a solar (or mildly enhanced above solar) value of $[\mathrm{C} / \mathrm{Fe}]$. The modest overabundance of the $\alpha$ elements of even atomic number $Z$, typical of halo turnoff stars, is confirmed in this example. The odd-Z element $\mathrm{P}$ is found to be somewhat deficient in HD 84937, at $[\mathrm{P} / \mathrm{Fe}]=-0.32$, which is again consistent with the handful of existing determinations for turnoff stars of such low metallicity. We show that the abundance of oxygen, deduced from the $\mathrm{OH}$ band from 3D computations, is not compatible with the abundance deduced from the red oxygen triplet. This incompatibility is explained by the existence of a chromosphere heating the shallow layers of the atmosphere where the $\mathrm{OH}$ band, in 3D computations, is mainly formed.

Conclusions. The abundance ratios are compared to the predictions of models of galactic nucleosynthesis and evolution.
\end{abstract}

Key words. ultraviolet: stars - stars: abundances - stars: chromospheres - Galaxy: halo - Galaxy: evolution - Galaxy: abundances

\section{Introduction}

The oldest surviving unevolved stars in the Milky Way are made from matter that is not very enriched in elements produced by previous (i.e. older, more massive) stars. The elements in these surviving stars show low abundances, but the observed deficiencies of individual elements are not identical. The measurement of these deviations is essential in order to constrain the different nucleosynthesis processes producing these elements. It is therefore very important to measure the detailed abundances for as many elements as possible, with the highest precision, in these very old stars. This paper is mainly devoted to the abundance of the light elements such as $\mathrm{P}, \mathrm{S}$, and $\mathrm{K}$, the evolution of which in the Galaxy is still poorly known.

HD 84937 is a bright, $(V=8.32$, Ducati 2002) very metalpoor, main-sequence turnoff star, often used as a reference

\footnotetext{
* Based on observations made with the NASA/ESA Hubble Space Telescope obtained under program GO-14161 at the Space Telescope Science Institute (STScI), which is operated by the Association of Universities for Research in Astronomy (AURA) and on observations collected at the European Organisation for Astronomical Research in the Southern Hemisphere (Archives of programmes 080.D-0347(A), 082.B-0610(A), 266.D-5655(A), and 073.D-0024(A) ).
}

for the field metal-poor stars (lately: Lawler et al. 2015), and consequently has recently been widely studied from spectra in the visible domain (Gehren et al. 2006; Lai et al. 2007; Mashonkina et al. 2011; Bergemann et al. 2012; Lind et al. 2013; VandenBerg et al. 2014; Sneden et al. 2016; Amarsi et al. 2016; Zhao et al. 2016). The model parameters are very similar in all these studies. The adopted effective temperature $T_{\text {eff }}$ ranges from 6300 to $6408 \mathrm{~K}$, and the metallicity $[\mathrm{Fe} / \mathrm{H}]^{1}$ from -1.9 (Lai et al. 2007) to -2.3 (Sneden et al. 2016). The surface gravity $\log g$ ranges from 4.0 to 4.1 in these studies; the only exception is a value of $\log g=4.5$ derived by Lai et al. (2007) from photometry rather than spectra.

Following VandenBerg et al. (2014), a comparison of the position of the star in the $M_{v}$ vs. $\log T_{\text {eff }}$ diagram with isochrones computed with different ages and metallicities, yields an age of $12.08 \pm 0.14 \mathrm{Gyr}$, adopting $T_{\mathrm{eff}}=6408 \mathrm{~K}$ and $\log g=4.05$. But this age depends critically on the adopted temperature. The error bar takes into account only the parallax uncertainty. If we instead adopt the parameters $T_{\text {eff }}=6300 \mathrm{~K}$ and $\log g=4.0$, as in Sneden et al. (2016), then the age of HD 84937 becomes

\footnotetext{
1 We adopt the classical notation that for element $\mathrm{X}: A(\mathrm{X})=$
} $\log \left(N_{\mathrm{X}} / N_{\mathrm{H}}\right)+12$ and $[\mathrm{X} / \mathrm{H}]=\log \left(N_{\mathrm{X}} / N_{\mathrm{H}}\right)_{\text {star }}-\log \left(N_{\mathrm{X}} / N_{\mathrm{H}}\right)_{\text {Sun }}$. 
$\approx 13.8 \mathrm{Gyr}$, very close to the age of the Universe: $13.799 \pm$ 0.038 Gyr (Planck Collaboration VII 2016). Unfortunately, a new parallax for this star is not yet available in the first Gaia release $^{2}$.

Owing to the weakness of spectral lines at the temperature and metallicity of HD 84937, the analysis of very high quality spectra is necessary to establish accurate abundances for this reference star. Taking advantage of a new UV high resolution spectrum from the Hubble Space Telescope (HST), complemented by previous high resolution, high signal-to-noise UVES and HARPS spectra obtained in the visible at the European Southern Observatory (Very Large and $3.6 \mathrm{~m}$ telescopes), we present new determinations of the abundances of several elements with atomic numbers $Z$ lying between $Z=6$ (carbon) and $Z=20$ (calcium). New abundances of $\mathrm{C}, \mathrm{Si}, \mathrm{P}$, and $\mathrm{S}$ in particular have been determined from the new HST spectrum, which contains many more useful lines of these elements than the optical and near-infrared spectra do in such weak-lined stars.

Phosphorus $(Z=15)$ has never been measured in HD 84937 and indeed has been measured in very few metalpoor stars, including only five stars with a metallicity below $[\mathrm{Fe} / \mathrm{H}]<-2.0$ dex (Caffau et al. 2011a, 2016; Roederer et al. 2014). Its behaviour hints at a primary origin in massive stars (Jacobson et al. 2014).

Sulphur $(Z=16)$ has never been measured in HD 84937 , but it has been measured in a sample of metal-poor stars (Caffau et al. 2005, 2016; Spite et al. 2011) from near-infrared lines (multiplets 1, 6, and 8) and in a carbon-rich metal-poor star (Roederer et al. 2016) from three UV lines (multiplet 2 uv) ${ }^{3}$. We present the first determination of the sulphur abundance in HD 84937, using the UV multiplet 1 uv compared to the infrared multiplet 1 .

For the first time the silicon abundance has been determined in HD 84937 from the profile of six UV lines and from the Si line in the visible.

\section{Observational data}

The HST spectrum was obtained as a legacy spectrum under GO14161, with R. Peterson as PI. As described further by Peterson (in prep.), this spectrum was acquired with the E230H grating of the STIS echelle spectrograph and the $0.2^{\prime \prime} \times 0.09^{\prime \prime}$ slit for a resolving power $R=114000$. Adopting five of the six E230H "prime" wavelength settings, with central wavelengths $201.3 \mathrm{~nm}, 226.3 \mathrm{~nm}, 251.3 \mathrm{~nm}, 276.2 \mathrm{~nm}$, and $301.3 \mathrm{~nm}$, resulted in an analysis coverage of $188.0-314.0 \mathrm{~nm}$. The total exposure time of $106.4 \mathrm{ks}(29.5 \mathrm{~h})$ yielded $S / N>=50$ per two-pixel resolution element at all wavelengths, except for the bluest and reddest.

To compare the abundances deduced from the HST spectra and the visible, we also used ground-based spectra from the ESO Very Large Telescope archives (spectrographs UVES and HARPS). For wavelengths shorter than $400 \mathrm{~nm}$ we used HARPS spectra. They have a resolving power $R=115000$ and a signal-to-noise per pixel $(\mathrm{S} / \mathrm{N})$ better than 150 per pixel. At longer wavelengths, in the blue we combined the individual UVES spectra to a mean UVES spectrum with a resolving power $R=65000$ and a $\mathrm{S} / \mathrm{N}$ per pixel better than 500. In the red, in order to minimise the influence of the telluric lines, and taking advantage of the fact that following Carney \& Latham (1987) and de Bruijne \& Eilers (2012), HD 84937 shows no detectable

\footnotetext{
2 http://wwW . cosmos. esa. int/web/gaia/dr1

With the multiplet numbering of Moore (1945).
}

velocity variability, we used three different mean spectra each obtained by combining individual spectra with almost the same geocentric radial velocity. Therefore, the positions of the telluric lines relative to the stellar lines are almost the same. These three mean spectra were obtained consecutively on a single night every year between 2002 and 2004. The resulting mean red spectra reach a resolving power of about 45000 and a $\mathrm{S} / \mathrm{N}$ per pixel equal to 310,510 , and 350 , respectively, in the region of the red C I lines.

\section{Spectral analysis and abundance measurements}

In our analysis we used OSMARCS model atmospheres (Gustafsson et al. 2008) together with the turbospectrum LTE synthesis code (Alvarez \& Plez 1998; Plez 2012). In this code, the collisional broadening by neutral hydrogen is generally computed following the theory developed by Anstee \& O'Mara (1991), Barklem et al. (2000) and Barklem \& O'Mara (2000).

For the computations we adopted the model parameters and $[\mathrm{Fe} / \mathrm{H}]$ derived for $\mathrm{HD} 84937$ in the same way as in Peterson \& Kurucz (2015) and Peterson (in prep.): $T_{\text {eff }}=6300 \mathrm{~K}, \log g=4.0$, and $v_{\mathrm{t}}=1.3 \mathrm{~km} \mathrm{~s}^{-1}$, along with the iron abundance they derived with these parameters, $[\mathrm{Fe} / \mathrm{H}]=-2.25$. These values deduced only from the UV spectra are in excellent agreement with the values derived for this star by Lawler et al. (2013) and Sneden et al. (2016) based on $V-K$ photometry and accurate HIPPARCos parallaxes.

The parameters and deduced abundance for each atomic line considered for this analysis are listed in Table 1. The mean abundances of the different elements are given in Table 2. For the computations of $[\mathrm{X} / \mathrm{H}]$ we adopted the solar abundances $A(\mathrm{C})=8.5^{4}, A(\mathrm{Mg})=7.54, A(\mathrm{Si})=7.52$, $A(\mathrm{P})=5.46, A(\mathrm{~S})=7.16, A(\mathrm{~K})=5.11$, and $A(\mathrm{Ca})=6.33$, following Caffau et al. (2011b) or Lodders et al. (2009).

We also provide 3D corrections for a selected number of atomic and molecular lines. They were computed from 3D synthetic spectra based on a CO ${ }^{5}$ BOLD (Freytag et al. 2012) model atmosphere from the Cosmological Impact of the First STars (CIFIST; Ludwig et al. 2009) grid, using the latest version of Linfor3D $^{5}$ (Gallagher et al. 2016a). A grid of 1D synthetic spectra - based on 1D LHD models (Caffau \& Ludwig 2007) computed with the same micro-physics as the 3D models - were fit to the $3 \mathrm{D}$ synthetic profiles to determine the $3 \mathrm{D}$ correction (see Gallagher et al. 2016b).

The 3D and equivalent 1D LHD models selected from the CIFIST grid have stellar parameters $T_{\text {eff }} / \log g /[\mathrm{Fe} / \mathrm{H}]=$ $6206 \mathrm{~K} / 4.0 /-2.0$, which is currently the model in the grid with stellar parameters closest to the adopted OSMARCS model parameters.

\subsection{Carbon and oxygen abundances}

In HD 84937 the $\mathrm{C}$ and $\mathrm{O}$ abundances can be derived from atomic lines and from the molecular $\mathrm{CH}$ and $\mathrm{OH}$ bands.

\subsection{1. $\mathrm{C}$ and $\mathrm{O}$ from atomic lines}

- Carbon: It is possible to determine the carbon abundance from a fit of the weak red C I lines near $910 \mathrm{~nm}$, the weak UV lines around 199 and $248 \mathrm{~nm}$ and the very strong UV

\footnotetext{
4 With the classical notation: $\mathrm{A}(X)=\log _{10}\left(N_{\mathrm{X}} / N_{\mathrm{H}}\right)+12$.

5 http://www.aip.de/Members/msteffen/linfor3d
} 
Table 1. Line-by-line 1D LTE abundances $A(\mathrm{X})$ from atomic lines.

\begin{tabular}{|c|c|c|c|c|c|}
\hline$Z$ & $\lambda(\mathrm{nm})$ & Ex. Pot. & $\log g f$ & $A(\mathrm{X})_{\mathrm{LTE}}$ & NLTEcorr \\
\hline \multirow[t]{9}{*}{6} & $\mathrm{C}(\mathrm{CI})$ & & & & \\
\hline & 193.0905 & 1.26 & -0.13 & 6.30 & $<0.01$ \\
\hline & 199.2012 & 1.26 & -5.74 & 6.47 & $<0.01$ \\
\hline & 199.3620 & 1.26 & -3.72 & 6.60 & 0.01 \\
\hline & 247.8561 & 2.68 & -0.96 & 6.28 & 0.01 \\
\hline & 906.2470 & 7.48 & -0.455 & 6.41 & -0.07 \\
\hline & 907.8280 & 7.48 & -0.581 & 6.43 & -0.08 \\
\hline & 908.8509 & 7.49 & -0.430 & 6.43 & -0.08 \\
\hline & 911.1799 & 7.49 & -0.297 & 6.49 & -0.10 \\
\hline \multirow[t]{4}{*}{8} & $\mathrm{O}$ & & & & \\
\hline & 777.1944 & 9.15 & 0.369 & 7.28 & -0.12 \\
\hline & 777.4166 & 9.15 & 0.223 & 7.28 & -0.12 \\
\hline & 777.5388 & 9.15 & 0.002 & 7.28 & -0.12 \\
\hline \multirow[t]{8}{*}{12} & $\mathrm{Mg}$ & & & & \\
\hline & 279.5528 & 0.00 & 0.100 & 5.55 & 0.00 \\
\hline & 280.2705 & 0.00 & -0.210 & 5.55 & 0.00 \\
\hline & 457.1096 & 0.00 & -5.623 & 5.61 & - \\
\hline & 470.2991 & 4.35 & -0.440 & 5.60 & 0.04 \\
\hline & 517.2684 & 2.71 & -0.380 & 5.59 & 0.06 \\
\hline & 518.3604 & 2.72 & -0.158 & 5.54 & 0.02 \\
\hline & 552.8405 & 4.34 & -0.341 & 5.49 & 0.01 \\
\hline \multirow[t]{9}{*}{14} & $\mathrm{Si}$ & & & & \\
\hline & 197.7598 & 0.00 & -1.309 & 5.66 & $<0.01$ \\
\hline & 198.0618 & 0.01 & -1.437 & 5.69 & $<0.01$ \\
\hline & 198.3233 & 0.01 & -1.192 & 5.65 & $<0.01$ \\
\hline & 208.2021 & 0.78 & -1.900 & 5.63 & -0.01 \\
\hline & 208.4462 & 0.78 & -1.880 & 5.62 & -0.01 \\
\hline & 212.2990 & 0.78 & -1.840 & 5.57 & 0.00 \\
\hline & 212.4122 & 0.78 & 0.149 & 5.40 & $<0.01$ \\
\hline & 390.5523 & 1.91 & -1.041 & 5.70 & 0.05 \\
\hline \multirow[t]{6}{*}{15} & $\mathrm{P}$ & & & & \\
\hline & 213.5469 & 1.41 & -1.240 & 2.98 & - \\
\hline & 213.6182 & 1.41 & -0.111 & 2.94 & - \\
\hline & 215.2939 & 1.41 & -0.357 & 2.81 & - \\
\hline & 253.3986 & 2.32 & -1.114 & 2.86 & - \\
\hline & 255.4911 & 2.33 & -1.231 & 2.85 & - \\
\hline \multirow[t]{7}{*}{16} & $\mathrm{~S}$ & & & & \\
\hline & 190.0287 & 0.00 & -3.709 & 5.30 & - \\
\hline & 191.4697 & 0.05 & -4.261 & 5.31 & - \\
\hline & 216.8884 & 1.14 & -3.962 & 5.30 & - \\
\hline & 921.2863 & 6.53 & 0.420 & 5.33 & -0.3 \\
\hline & 922.8093 & 6.53 & 0.260 & 5.49 & -0.3 \\
\hline & 923.7538 & 6.53 & 0.040 & 5.40 & -0.3 \\
\hline \multirow[t]{3}{*}{19} & $\mathrm{~K}$ & & & & \\
\hline & 766.4899 & 0.00 & 0.149 & 3.46 & -0.2 \\
\hline & 769.8964 & 0.00 & -0.154 & 3.40 & -0.2 \\
\hline \multirow[t]{17}{*}{20} & $\mathrm{Ca}$ & & & & \\
\hline & 428.3011 & 1.89 & -0.220 & 4.55 & 0.07 \\
\hline & 431.8652 & 1.90 & -0.210 & 4.49 & 0.06 \\
\hline & 442.5437 & 1.88 & -0.360 & 4.50 & 0.07 \\
\hline & 443.5679 & 1.89 & -0.520 & 4.52 & 0.07 \\
\hline & 445.4779 & 1.90 & 0.260 & 4.50 & 0.04 \\
\hline & 526.5556 & 2.52 & -0.260 & 4.63 & 0.13 \\
\hline & 534.9465 & 2.71 & -0.310 & 4.50 & 0.12 \\
\hline & 558.1965 & 2.52 & -0.710 & 4.67 & 0.09 \\
\hline & 558.8749 & 2.52 & 0.210 & 4.62 & 0.08 \\
\hline & 559.0114 & 2.52 & -0.710 & 4.67 & 0.09 \\
\hline & 560.1277 & 2.52 & -0.690 & 4.69 & 0.09 \\
\hline & 585.7451 & 2.93 & 0.230 & 4.50 & 0.08 \\
\hline & 610.2723 & 1.88 & -0.790 & 4.51 & 0.05 \\
\hline & 612.2217 & 1.89 & -0.320 & 4.52 & 0.00 \\
\hline & 616.2173 & 1.90 & -0.090 & 4.54 & 0.00 \\
\hline & 643.9075 & 2.52 & 0.470 & 4.42 & -0.02 \\
\hline
\end{tabular}

Notes. The adopted non-LTE corrections to be applied are given in the last column. For S, the non-LTE correction includes the 3D correction. Air wavelengths are given for wavelengths $>200 \mathrm{~nm}$ and vacuum values below.
Table 2. Mean abundances of the different elements.

\begin{tabular}{|c|c|c|c|c|}
\hline Element & $\begin{array}{c}{[\mathrm{X} / \mathrm{H}]} \\
1 \mathrm{D} \\
\text { LTE }\end{array}$ & $\begin{array}{c}{[\mathrm{X} / \mathrm{Fe}]} \\
1 \mathrm{D} \\
\mathrm{LTE}\end{array}$ & $\sigma$ & $\begin{array}{c}{[\mathrm{X} / \mathrm{Fe}]} \\
\text { corrected } \\
\text { (adopted) }\end{array}$ \\
\hline $\mathrm{C}(\mathrm{CH})$ & -1.86 & +0.39 & - & +0.11 \\
\hline $\mathrm{C}(\mathrm{CI})(\mathrm{UV})$ & -2.09 & +0.16 & 0.15 & +0.17 \\
\hline $\mathrm{C}(\mathrm{C} \mathrm{I})(\mathrm{red})$ & -2.06 & +0.19 & 0.04 & +0.10 \\
\hline $\mathrm{O}(\mathrm{OH})$ & -1.47 & +0.78 & & \\
\hline $\mathrm{O}(\mathrm{OI})(\mathrm{red})$ & -1.48 & +0.77 & - & +0.65 \\
\hline $\mathrm{Mg}$ & -1.99 & +0.26 & 0.07 & +0.30 \\
\hline $\mathrm{Si}$ & -1.90 & +0.35 & 0.06 & +0.38 \\
\hline $\mathrm{P}$ & -2.57 & -0.32 & 0.07 & -0.32 \\
\hline S (UV & -1.83 & +0.42 & 0.03 & +0.42 \\
\hline$S$ (red) & -1.75 & +0.50 & 0.08 & +0.20 \\
\hline $\mathrm{K}$ (red) & -1.68 & +0.57 & 0.03 & +0.37 \\
\hline $\mathrm{Ca}$ & -1.78 & +0.47 & 0.08 & +0.53 \\
\hline
\end{tabular}

Notes. In Cols. 2 and 3 are given the abundances of the different elements derived from the 1D computations. We adopted the solar abundances given in section 3 and $[\mathrm{Fe} / \mathrm{H}]=-2.25$. In the last column is given $[\mathrm{X} / \mathrm{Fe}]$ corrected for NLTE and $3 \mathrm{D}$ when possible.

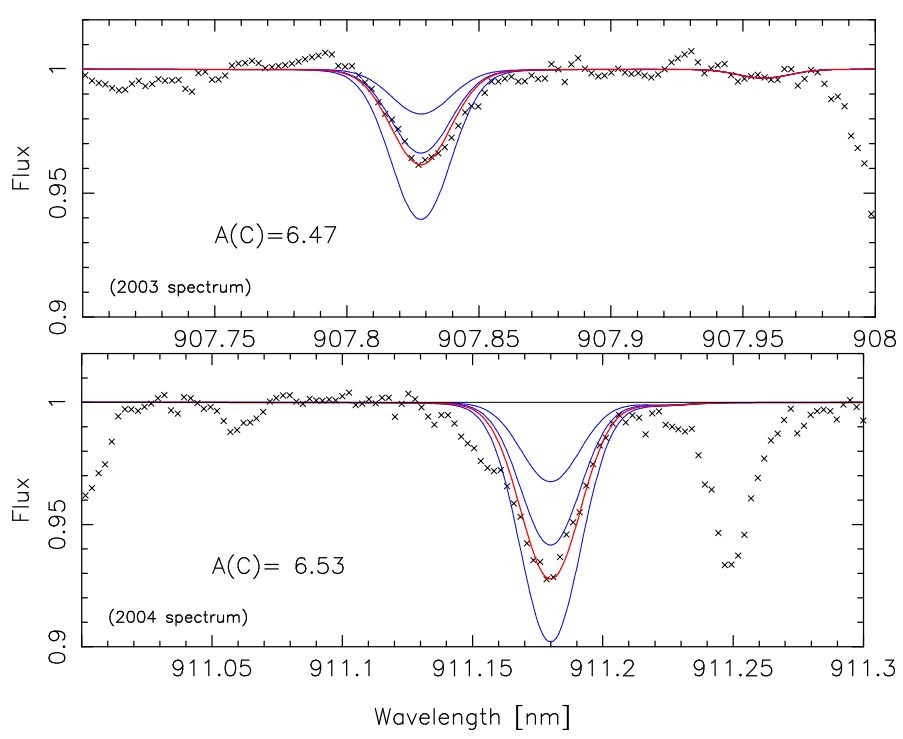

Fig. 1. Examples of the best fits (red thick line) of two atomic lines of $\mathrm{C} \mathrm{I}$ in the red. Synthetic profiles (blue thin lines) have been computed with $A(\mathrm{C})=6.1,6.4$, and 6.7 .

C I line at $193 \mathrm{~nm}$. In the three cases we estimate (Table 2) that the error in $[\mathrm{C} / \mathrm{H}]$ is $\approx 0.1 \mathrm{dex}$.

The red $\mathrm{CI}$ lines are located within a $\mathrm{H}_{2} \mathrm{O}$ telluric band. In this region we had several spectra observed at different dates $(2002,2003,2004)$, and for each mean spectrum the relative position of the telluric lines was different. Thus we chose to measure each C I line from only those spectra in which telluric contamination was minimal. The 906.25 line was measured only on the mean 2003 spectrum, the 907.83 line could be measured on the 2002 and 2003 mean spectra, 


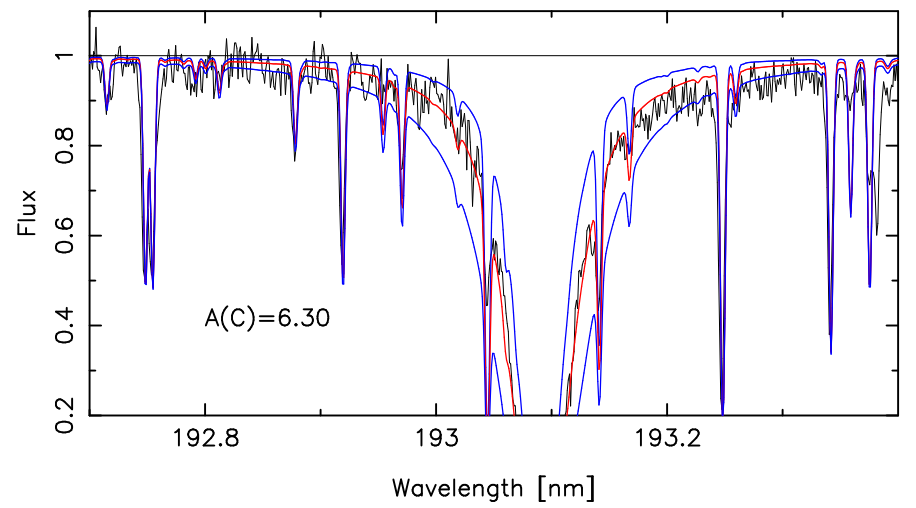

Fig. 2. Observed profile of the strong C I line in HD 84937. The blue thin lines represent the synthetic profiles computed with $\mathrm{A}(\mathrm{C})=6.0$ and 6.9 . The thick red line represents the best fit (1D computations) $A(C)=6.3$.

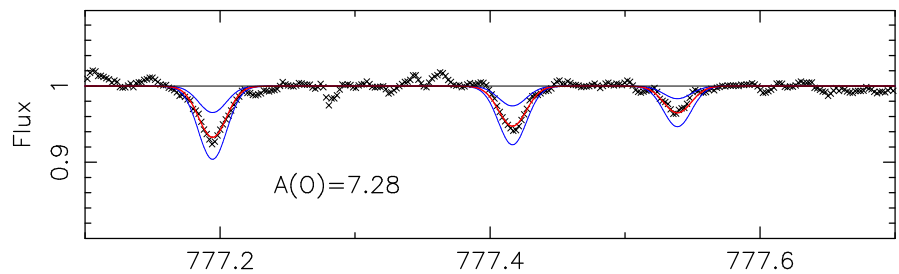

Fig. 3. Comparison between the observed and synthetic profiles of the red oxygen triplet in HD 84937. The synthetic profiles (blue thin lines) of the oxygen triplet have been computed with $\mathrm{A}(\mathrm{O})=6.9$ and 7.5. The thick red line represents the best fit for $\mathrm{A}(\mathrm{O})=7.28$.

and the 911.18 line only on the 2004 spectrum. An example of the fit is given in Fig. 1. In Fig. 2 we present the fit of the wings of the strong $\mathrm{C}$ I line at $193.1 \mathrm{~nm}$.

Fabbian et al. (2006, 2009) computed a non-LTE correction for HD 84937 of -0.2 dex for the high excitation C I red lines. Zhao et al. (2016) and Mashonkina (priv. comm.) computed a correction close to -0.1 dex for the red $\mathrm{C} I$ lines and $\leq 0.01$ dex for the UV C I lines; we adopted these corrections (Table 1). These new measurements point to a $[\mathrm{C} / \mathrm{Fe}]$ ratio in HD 84937 close to solar (Table 2).

- Oxygen We derived the oxygen abundance in HD 84937 from the oxygen triplet at $777 \mathrm{~nm}$ (Fig. 3). The non-LTE correction $(-0.12 \mathrm{dex})$ was adopted from the calculations of Zhao et al. (2016) and leads to $[\mathrm{O} / \mathrm{H}]=7.16 \pm 0.1$. The resulting value $[\mathrm{O} / \mathrm{Fe}]=+0.65$ is in good agreement with the value obtained from the forbidden [O I] line in the EMP giants by Spite et al. (2005), and is in a reasonable agreement, inside the measurement errors, with the value obtained by Zhao et al. (2016) from the red O I lines (part of the difference indeed comes from the adopted $[\mathrm{Fe} / \mathrm{H}]$ value).

\subsection{2. $\mathrm{C}$ and $\mathrm{O}$ from $\mathrm{CH}$ and $\mathrm{OH}$ molecular bands}

\section{- 1D Computations}

- We computed the $\mathrm{CH}$ carbon abundance by fitting the profile of the $\mathrm{CH}$ feature at $314.3 \mathrm{~nm}$ and of the $G$-band between 430.8 and $431.4 \mathrm{~nm}$. Line lists for ${ }^{12} \mathrm{CH}$ and ${ }^{13} \mathrm{CH}$ (Masseron et al. 2014) were included in the synthesis. The result is presented in Fig. 4. For the UV feature the best fit corresponds to $[\mathrm{C} / \mathrm{H}]=-1.90 \mathrm{dex}$, i.e., $[\mathrm{C} / \mathrm{Fe}]=+0.35 \mathrm{dex}$, and for the $G$-band to $[\mathrm{C} / \mathrm{H}]=-1.86$ dex, i.e., $[\mathrm{C} / \mathrm{Fe}]=+0.39$ dex. The $\mathrm{CH}$ band in HD 84937 is very weak and thus the
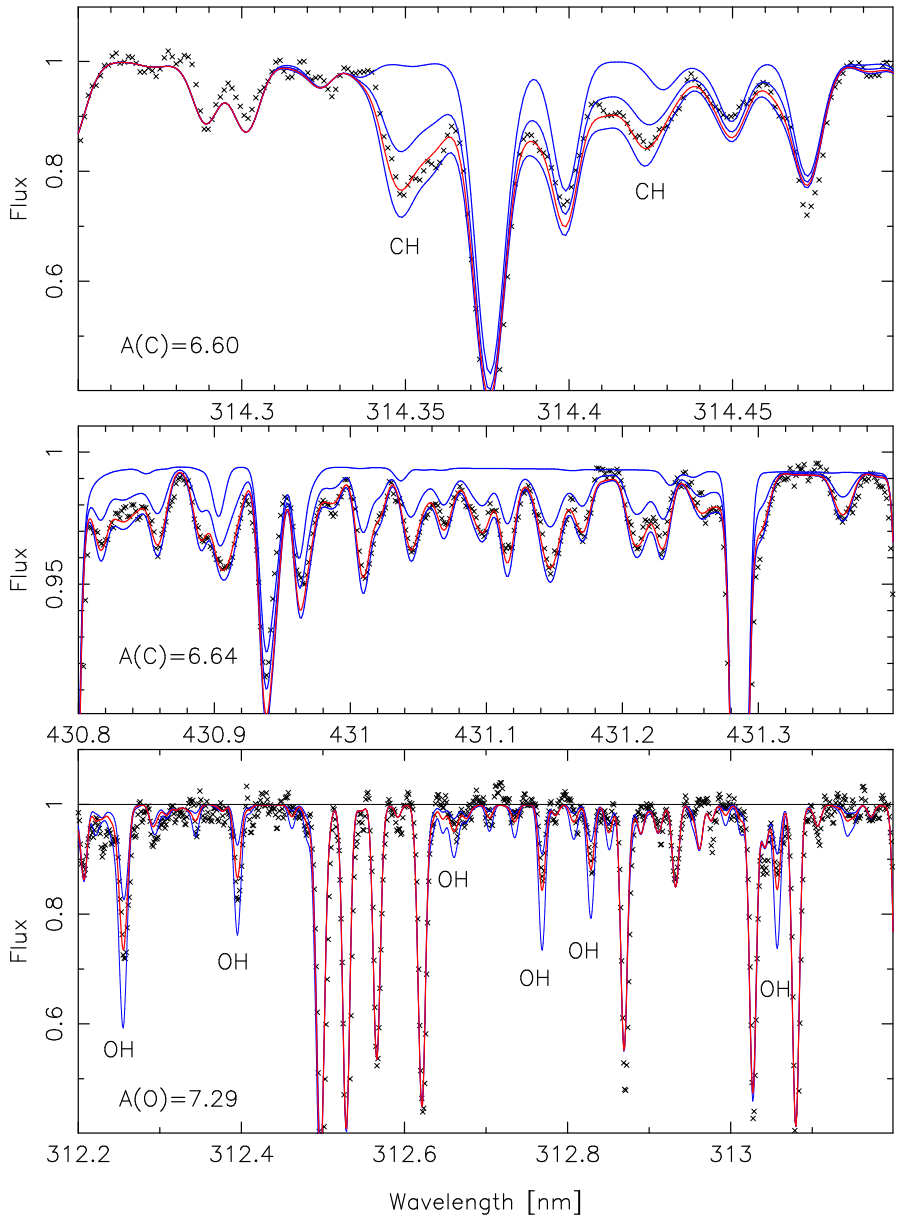

Fig. 4. Observed profile of the $\mathrm{CH}$ and $\mathrm{OH}$ bands in HD 84937. For the $\mathrm{CH}$ bands the 1D ETL synthetic profiles (blue thin lines) have been computed with $A(\mathrm{C})=0.0,6.4$, and 6.7 . The absorption by the wing of $\mathrm{H} \gamma$ has been included in the computation of the $G$-band. The thick red line represents the best fit: $A(\mathrm{C})=6.60$ for the UV CH feature and $A(\mathrm{C})=6.64$ for the $G$-band. The synthetic profile of the $\mathrm{OH}$ band has been computed with $A(\mathrm{O})=7.0$ and 7.6. The best fit is obtained with $A(\mathrm{O})=7.29$

corresponding abundance is very sensitive to the position of the continuum and in this case the error on $[\mathrm{C} / \mathrm{H}]$ is estimated to $\approx 0.15$ dex.

- A fit of the $\mathrm{OH}$ band between 312 and $314 \mathrm{~nm}$ leads to $[\mathrm{O} / \mathrm{H}]=-1.47$ (Fig. 4) and $[\mathrm{O} / \mathrm{Fe}]=+0.78$.

There is, in HD 84937, a rather good agreement between the abundances deduced from the atomic lines (Table 2) and the molecular bands from 1D computations. However, in metal-poor stars the molecular lines are sensitive to convective effects, neglected in the 1D computations, and we have to take them into account.

\section{- 3D Computations}

We computed 3D corrections to the 1D abundances determined for the $G$-band, and $14 \mathrm{OH}$ lines in the wavelength range $312.24-312.83 \mathrm{~nm}$. We define a $3 \mathrm{D}$ correction, $\Delta_{3 \mathrm{D}}$, with the formalism given by Gallagher et al. (2016b), i.e., $\Delta_{3 \mathrm{D}}=A(\mathrm{X})_{3 \mathrm{D}}-A(\mathrm{X})_{1 \mathrm{D}}$, where the $1 \mathrm{D}$ synthesis is computed from LHD model atmospheres. We computed 3D profiles of $\mathrm{CH}$ and $\mathrm{OH}$ using the absolute carbon and oxygen abundances derived from the 1D non-LTE computations of the 

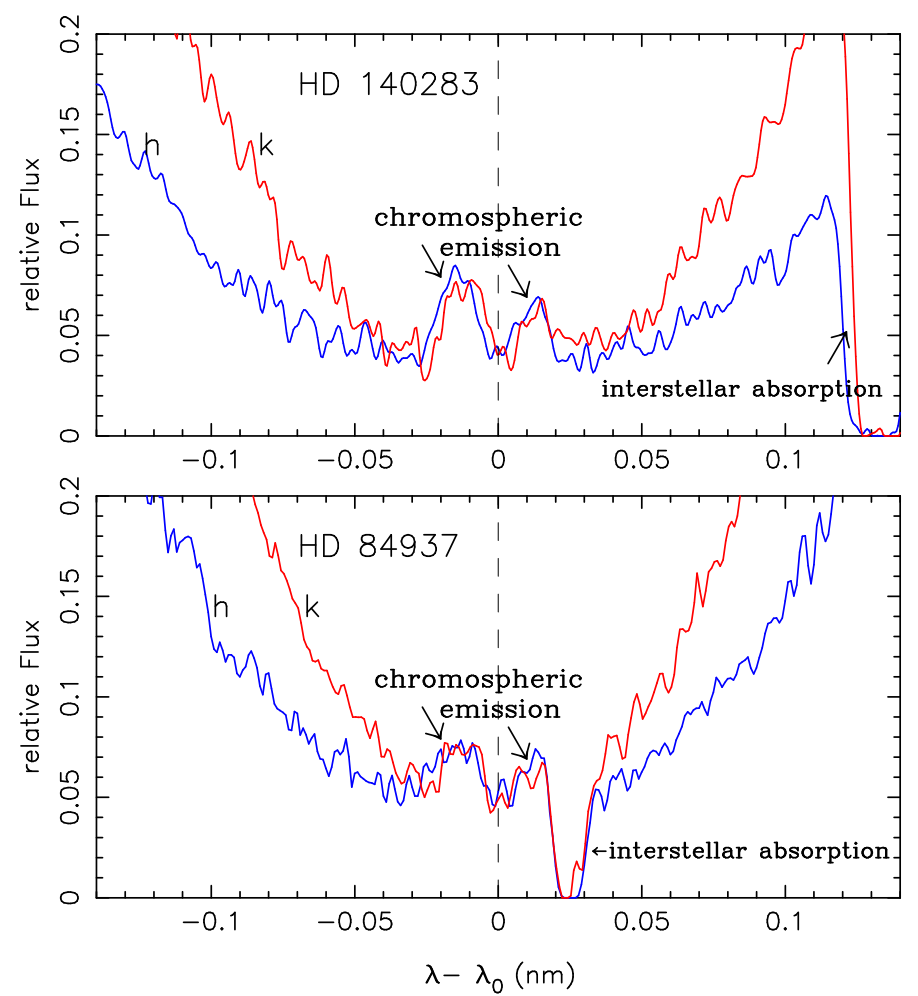

Fig. 5. Centre of the the $h$ and $k \mathrm{Mg}$ II lines in the HST/STIS UV spectra of HD 84937 and HD 140283. The profiles of the $h$ and $k$ lines have been overplotted to show the emission at the centre of both lines indicating the existence of a chromosphere. In HD 84937 an interstellar line, with a radial velocity close to the radial velocity of the star, perturbs the profile on the red side of the core.

red $\mathrm{C}$ I and $\mathrm{O}$ I atomic lines and thus with a $\mathrm{C} / \mathrm{O}$ ratio of $\approx 0.15$. (Unlike the $1 \mathrm{D}$ computations, the $3 \mathrm{D}$ computations are sensitive to the $\mathrm{C} / \mathrm{O}$ ratio $)^{6}$.

For the $G$-band we found a $3 \mathrm{D}$ correction of -0.28 dex. The resulting $\mathrm{C}$ abundance, $[\mathrm{C} / \mathrm{H}]=-2.14$ or $[\mathrm{C} / \mathrm{Fe}]=+0.11$, is in very good agreement with the abundance deduced from the atomic lines (Table 2). However, the $\mathrm{OH}$ lines were far stronger in $3 \mathrm{D}$ than in $1 \mathrm{D}$. In fact, owing to the low $\mathrm{C} / \mathrm{O}$ ratio in the atmosphere of a typical metal-poor star like HD 84937, the $\mathrm{OH}$ lines are mainly formed in the most external regions of the atmosphere, which are about $300 \mathrm{~K}$ cooler in the 3D model than in the 1D model (see Fig. 1 in Gallagher et al. 2016b, for a metallicity of -2 ). In this case, the 3D correction reaches -0.72 dex. Applying this $3 \mathrm{D}$ correction to the $\mathrm{OH}$ band would lead to a value of $[\mathrm{O} / \mathrm{H}]=-2.19([\mathrm{O} / \mathrm{Fe}]=+0.06)$, a value hardly compatible with the observation of the atomic lines. For HD 84937 the adopted 3D model seems to be too cool in the most outer layers. This could be due to the omission of a hot solar-like chromosphere in our 3D model.

From HST spectra of the Mg II lines near $280 \mathrm{~nm}$, Peterson \& Schrijver (1997) showed that solar-like chromospheres routinely occur among turnoff metal-poor stars despite their relatively low degree of activity. From seven such stars of a wide metallicity range down to $1 / 300$ solar whose radial velocities largely shifted the line cores away from the interstellar absorption, this work demonstrated that double-peaked emission cores were always present, and that the blue peak was usually stronger than the red as is also the usual case for the quiet Sun.

\footnotetext{
$6 \quad \mathrm{X} / \mathrm{Y}=N(\mathrm{X}) / N(\mathrm{Y})=10^{\left[A(\mathrm{X})_{*}-A(\mathrm{Y})_{*}\right]}$.
}

They concluded that "while these data do not rule out magnetic fields they support an acoustic origin of chromospheric emission and show that relatively inactive solar-type stars of all ages have chromospheres whose characteristics are largely independent of metallicity". Subsequent work, based on observations of He I $1083 \mathrm{~nm}$ absorption features, have confirmed these results (e.g., Takeda \& Takada-Hidai 2011). More work on this subject, based on the profiles of the Mg II lines in HST/STIS spectra of several metal-poor stars, is ongoing (Peterson \& Tarbell, in prep.). In Fig. 5 we present the profile of the $\mathrm{Mg}$ II $h$ and $k$ lines in our HST/STIS spectrum of HD 84937 and in the G0-14161 E230H spectrum of another classical extremely metal-poor star, HD 140283 (Peterson, in prep.). In HD 140283 the two peaks typical of a solar-like chromosphere - are clearly visible; unfortunately, in HD 84937 an interstellar line with a radial velocity very close to the radial velocity of the star perturbs this profile on the red side, and the red peak of the emission is not clearly visible.

We also computed the 3D correction for two CI lines: the very strong line at $193.09 \mathrm{~nm}$ (Fig. 2) and the line at $199.36 \mathrm{~nm}$. In both cases we found a correction lower than the measurement error (respectively +0.06 and -0.07 ).

\subsection{Abundances of classical $\alpha$ elements: magnesium and calcium}

For a more homogeneous comparison, in the visible we derived the magnesium and calcium abundances from the equivalent widths of a set of lines already used in extremely metal-poor (EMP) main-sequence turnoff stars by Bonifacio et al. (2009).

For the optical Mg I lines in Table 1, we adopted the non-LTE correction computed by Zhao et al. (2016) and we found a mild overabundance of $\mathrm{Mg}$ (Table $\left.2:[\mathrm{Mg} / \mathrm{Fe}]_{\text {LTE }}=+0.30 \mathrm{dex}\right)$, as did Gehren et al. (2006) and Zhao et al. (2016). This value is also in good agreement with the value we deduce from the wings of the very strong $\mathrm{Mg}$ II $\mathrm{h}$ and $\mathrm{k}$ lines around $280 \mathrm{~nm}\left([\mathrm{Mg} / \mathrm{Fe}]_{\mathrm{LTE}}=\right.$ $+0.26 \mathrm{dex})$. The non-LTE correction has not been computed for these UV lines. However, following Abia \& Mashonkina (2004), since $\mathrm{Mg}$ II is the majority species, departures from LTE are expected to be caused mainly by radiative $b-b$ transitions, and no process seems to affect the Mg II ground-state population. As a consequence, the non-LTE correction for the resonance $h$ and $k$ $\mathrm{Mg}$ II lines should be negligible.

Our LTE calcium abundances in Table 1 have been corrected for non-LTE effects following Spite et al. (2012). We have three lines in common with Zhao et al. (2016), and the non-LTE corrections for these lines do not differ by more than 0.02 dex. The Ca abundance found in HD 84937 is in very good agreement with the mean abundances obtained, using the same set of lines, for EMP turnoff stars with $-3.6<[\mathrm{Fe} / \mathrm{H}]<-2.5$ (Bonifacio et al. 2009; Spite et al. 2012).

\subsection{Silicon abundance}

The silicon abundance could be deduced from seven silicon lines in the UV and another one at $390 \mathrm{~nm}$. This last line was used to determine the $\mathrm{Si}$ abundance in the sample of EMP turnoff stars studied in Bonifacio et al. (2009). Shi et al. (2009) computed for this line a non-LTE correction of +0.05 dex. The non-LTE correction for the ultraviolet lines were computed by Mashonkina (priv. comm.) and are less than 0.1 dex (Table 1). Our derived overabundance of $\mathrm{Si}$ is then $[\mathrm{Si} / \mathrm{H}]=-1.87 \pm 0.10$ or $[\mathrm{Si} / \mathrm{Fe}]=+0.38$. It is interesting to note that this value is higher 

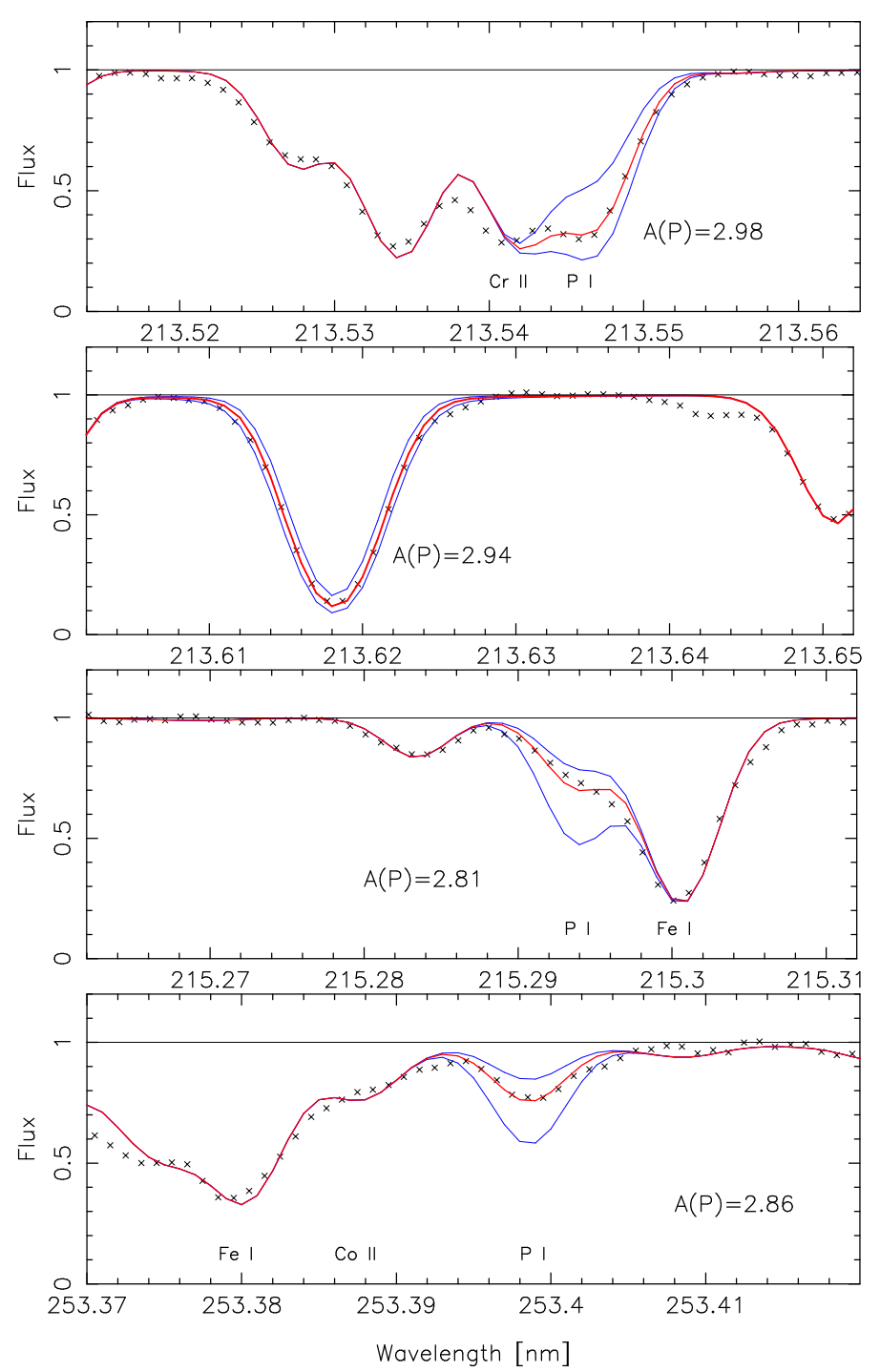

Fig. 6. Comparison of the observed (crosses) and synthetic (curves) spectra in HD 84937 in the region of the four P I lines analysed in this work. The red thick line marks the best-fit synthetic spectrum, obtained with the value of $A(\mathrm{P})$ indicated in the figure. The thin blue lines show synthetic spectra computed with $A(\mathrm{P})=2.6$ and $A(\mathrm{P})=3.2$. As is usually done, the $\log g f$ values of the blending lines have been adjusted to give a more correct representation of the different features.

than the mean silicon overabundance derived in EMP dwarfs from the $390 \mathrm{~nm}$ line alone: $[\mathrm{Si} / \mathrm{Fe}] \approx+0.09 \pm 0.14$. But this mean value has not been corrected for non-LTE effects and, in these EMP turnoff stars with $[\mathrm{Fe} / \mathrm{H}] \leq-3$, the non-LTE correction could reach 0.25 dex (Shi et al. 2009). As a consequence the silicon abundance found in the UV in HD 84937 is compatible with the results of Bonifacio et al. (2009). As for $\mathrm{Mg}$ and $\mathrm{Ca}$, our $[\mathrm{Si} / \mathrm{H}]$ value is very close to the value found by Zhao et al. (2016), and the small difference in $[\mathrm{Si} / \mathrm{Fe}]$ is attributed to the adopted $[\mathrm{Fe} / \mathrm{H}]$ values.

\subsection{Phosphorus abundance}

The phosphorus abundance is derived from five ultraviolet lines of the multiplets 4(uv), 8(uv), and 9(uv), leading to $[\mathrm{P} / \mathrm{Fe}]=-0.32$. The statistical error reaches 0.07 dex. Some other $\mathrm{P}$ lines are visible in the spectrum, but these lines are so severely blended that they cannot be used to determine the
P abundance with precision. In Fig. 6 we show the fit of the observed lines with the computed spectra. The value of $[\mathrm{P} / \mathrm{Fe}]$ in HD 84937 confirms the low value of $[\mathrm{P} / \mathrm{Fe}]$ in metal-poor stars (Fig. 7).

Recently R. Kurucz has published new $\log g f$ values for the $\mathrm{P}$ lines in the UV and in the $\mathrm{red}^{7}$. These new values are about 0.1 dex higher than the old ones. Consequently, their adoption here would reduce the $[\mathrm{P} / \mathrm{Fe}]$ values of Tables 1 and 2 by about 0.1 dex.

To date, no non-LTE calculations have been performed for PI features; following Jacobson et al. (2014) they are expected to be small in this type of star.

\subsection{Sulphur abundance}

In HD 84937, the sulphur abundance could be derived from the profile of two UV features at 190.03 and $191.47 \mathrm{~nm}$ and from the profiles of the red multiplet 1 (Table 2). This multiplet is contaminated by a telluric band of $\mathrm{H}_{2} \mathrm{O}$. We measured the $\mathrm{S}$ lines only on spectra where they were not blended by a telluric line. The 921.2 line could be measured on the 2002, 2003, and 2004 spectra, but the other two lines ( 922.8 and $923.7 \mathrm{~m}$ ) only on the 2002 and 2003 spectra. We obtained good agreement between the abundances deduced from the two different sets of lines: $[\mathrm{S} / \mathrm{Fe}]=+0.42$ dex from the UV lines and $[\mathrm{S} / \mathrm{Fe}]=+0.50$ from the red lines.

It is well known that non-LTE effects influence the profiles of the red multiplet 1 . Following Takeda et al. (2005), the nonLTE correction is about -0.2 dex for the model parameters of HD 84937. However, following Spite et al. (2011), based on a new model atom of sulphur by Korotin $(2008,2009 a, b)$ and new photoionisation rates, the NLTE correction for the model of HD 84937 reaches -0.4 dex. On the other hand, it has been computed that in the case of this multiplet, the $3 \mathrm{D}$ correction is not negligible and reaches +0.1 dex (Spite et al. 2011). This small but positive correction has been added to the NLTE correction. Finally, we find from the red multiplet $1,[\mathrm{~S} / \mathrm{Fe}]=+0.20 \mathrm{dex}$.

As far as we are aware, non-LTE corrections of the UV features have never been computed, but they are probably as small as for $\mathrm{C}$ and $\mathrm{Si}$. We estimate a sulphur overabundance in $\mathrm{HD} 84937$ of $[\mathrm{S} / \mathrm{Fe}] \approx 0.3 \mathrm{dex}$, in good agreement with the mean sulphur overabundance found in mid metal-poor stars (François 1986) and in EMP stars (Spite et al. 2011).

\subsection{Potassium abundance}

The abundance of $\mathrm{K}$ has been deduced from the red lines at $766.4899 \mathrm{~nm}$ and $769.8964 \mathrm{~nm}$. Following Takeda et al. (2002), the non-LTE correction for the model parameters of HD 84937 reaches -0.3 dex, but Zhao et al. (2016) has computed a correction of -0.2 dex and we adopted this latter value. As a consequence we find for this star a ratio $[\mathrm{K} / \mathrm{Fe}]=0.37 \pm 0.10$, in excellent agreement with the value found for the EMP stars by Takeda et al. (2009) and Andrievsky et al. (2010).

\section{Discussion and conclusion}

Our analysis of high quality spectra for HD 84937, notably the HST ultraviolet spectra as well as ground-based optical/nearinfrared spectra, has allowed us to refine the abundances of several light elements between $\mathrm{C}$ and $\mathrm{Ca}$ in this star, and provide the first measurements of its $\mathrm{P}, \mathrm{S}$, and $\mathrm{K}$ abundances and a more

7 http://kurucz.harvard. edu/linelists.html see file GFALL. 
firmly established Si abundance. Accurate abundances of such key elements are vital in reference stars like HD 84937 in order to better interpret chemical evolution models and supernovae enrichment in the Galactic halo.

The presence of emission-core reversal at the centre of the very strong $\mathrm{Mg}$ II resonance lines confirms the existence of a chromosphere around HD 84937 (Peterson \& Schrijver 1997).

The abundances of $\mathrm{C}$ and $\mathrm{O}$ deduced from the atomic lines are in good agreement with the abundances obtained in the EMP stars (Bonifacio et al. 2009; Spite et al. 2005).

The $\mathrm{C}$ abundance deduced from the $\mathrm{CH}$ molecular band after the $3 \mathrm{D}-1 \mathrm{D}$ correction is in very good agreement with the abundance deduced from the atomic lines (Table 2).

The oxygen abundance deduced from the $\mathrm{OH}$ molecular band computed with 1D models agree with the abundances deduced from the atomic lines; however, when 3D computations are done, the abundance of oxygen deduced from the $\mathrm{OH}$ band is incompatible with the abundance deduced from the atomic lines. In 3D computations, at variance with $1 \mathrm{D}$ computations, the $\mathrm{OH}$ band is formed predominantly in shallow atmospheric layers, and since 3D models are cooler at the surface than 1D models, $\mathrm{OH}$ lines are highly strengthened. However, current 3D models for metal-poor turnoff stars do not include the rise in temperature exhibited by a solar-like chromosphere. These models have been built only for the Sun, but similar models would be useful in order to analyse molecular features in classical metal-poor turnoff stars.

The classical $\alpha$ elements $\mathrm{Mg}, \mathrm{Si}$, and $\mathrm{Ca}$ have about the same overabundance in HD 84937 as observed in the EMP turnoff stars studied in Bonifacio et al. (2009). HD 84937 is also mildly enhanced in $\mathrm{K}$, as are the other metal-poor stars.

- The ratio $[\mathrm{P} / \mathrm{Fe}]$ is found to be slightly below the solar value, as is typically seen in very metal-poor stars (Fig. 7) and more generally for the light odd-Z elements like $\mathrm{Na}$ and $\mathrm{Al}$. Molaro et al. (2001) also measured [P/Fe] in a dust free Ly- $\alpha$ system that has the same metallicity as HD 84937, and they found $[\mathrm{P} / \mathrm{Fe}]=-0.27$, about the same ratio as observed in HD 84937 (Table 2).

In Fig. 7 we show the $\mathrm{P}$ measurements of Roederer et al. (2014, 2016). The two most metal-poor stars in the figure are $\mathrm{BD}+44^{\circ} 493([\mathrm{Fe} / \mathrm{H}]=-3.88)$ and $\mathrm{G} \mathrm{64}-12,([\mathrm{Fe} / \mathrm{H}]=$ -3.28). They are both carbon-enhanced metal-poor stars (CEMP) following Roederer et al. (2016) and Placco et al. (2016), but in these stars the neutron-capture elements are not enhanced. They are "CEMP-no" stars and thus the composition of their atmosphere is supposed to reflect the abundance of the cloud that formed the star. This cloud would have been enriched by a faint supernova $(\mathrm{SN})$ providing carbon and the lighter elements, but the elements heavier than $\mathrm{Mg}$ are not affected (see, e.g., Bonifacio et al. 2015). As a consequence, the $\mathrm{P}$ abundance in these stars should be comparable to the $\mathrm{P}$ abundance in classical metal-poor stars (i.e., metal-poor stars without $\mathrm{C}$ enhancement) and we include them in the figure.

According to Woosley \& Weaver (1995) and West \& Heger (2013), the isotope ${ }^{31} \mathrm{P}$ is produced mostly in SN II in both $\mathrm{O}$ and $\mathrm{Ne}$ burning shells, and its ejection is not very sensitive to the explosion mechanism. Taking into account core-collapse supernovae and hypernovae, Kobayashi et al. (2006, 2011) produced models of chemical evolution for the halo. We overplotted their best model to the very few available $\mathrm{P}$ data points (Fig. 7). Also plotted in this figure are the metallicity-dependent model of Timmes et al. (1995),

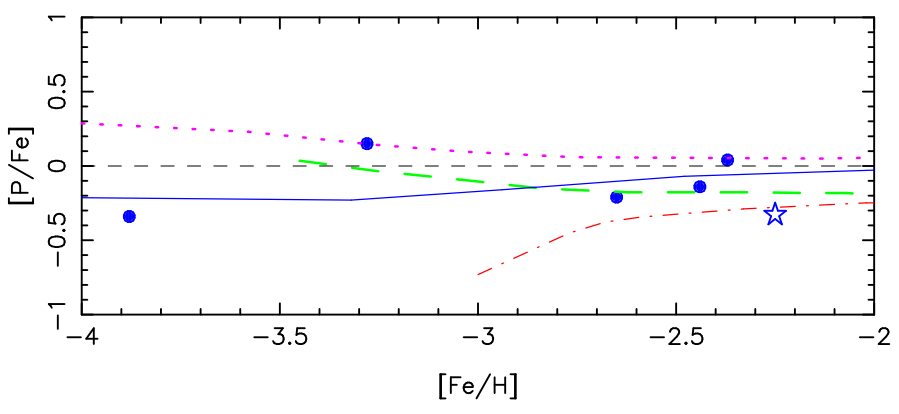

Fig. 7. $[\mathrm{P} / \mathrm{Fe}]$ vs. $[\mathrm{Fe} / \mathrm{H}]$ is shown for the five stars analysed by Roederer et al. (2014) and Roederer et al. (2016) with $[\mathrm{Fe} / \mathrm{H}]<-2.0$ (blue dots), plus our result for HD 84937 (blue star symbol). The theoretical predictions of Timmes et al. (1995; red dot-dashed line), Goswami \& Prantzos (2000; pink dotted line), Kobayashi et al. (2011; blue thick line), and Cescutti et al. (2012; green dashed line) are also represented.

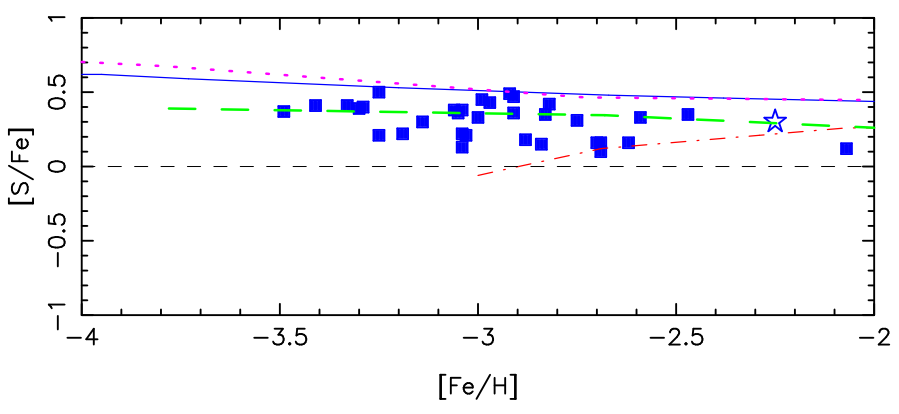

Fig. 8. $[\mathrm{S} / \mathrm{Fe}]$ vs. $[\mathrm{Fe} / \mathrm{H}]$ for metal-poor stars with $[\mathrm{Fe} / \mathrm{H}]<-2.0$ (blue squares), plus our result for HD 84937 (blue star symbol). The theoretical predictions of Timmes et al. (1995; red dot-dashed line), Goswami \& Prantzos (2000; pink dotted line), Kobayashi et al. (2011; blue thick line), and Matteucci (2016; green dashed line) are also represented.

the model of Goswami \& Prantzos (2000) using yields from Woosley \& Weaver (1995) at variable metallicities, and model 4 of Cescutti et al. (2012). The best fit seems to be obtained with the Kobayashi et al. (2011) model. However in the interpretation of the abundance trends we have to be very prudent. We note that NLTE computations have not been done for this element and that following Caffau et al. (2016) for the disk stars there is a shift of 0.23 dex between the UVHST and the near-infrared measurements of the P abundance (see their Fig. 5). This disagreement could be the result of different non-LTE corrections for the UV and near-infrared lines but could be also the result of systematic errors in the $g f$ values.

- Sulphur is an $\alpha$ element mainly produced in massive supernovae or hypernovae. In Fig. 8 we compare the observed $[\mathrm{S} / \mathrm{Fe}]$ ratios to the abundance ratios predicted by the models of Goswami \& Prantzos (2000), Kobayashi et al. (2011) and Matteucci (2016). The best fit is obtained with the model of Matteucci (2016).

- In the very metal-poor stars, potassium (an odd element: $Z=19$ ) is enhanced relative to iron (Fig. 9) as are the (even) $\alpha$ elements. The models struggle to represent the abundance of $\mathrm{K}$ in the Galactic halo. The best fit (Fig. 9) is achieved by the model of Goswami \& Prantzos (2000); however, these authors point out that this good representation could be fortuitous and might be due to a factor of two reduction of the Fe yields combined with the adopted IMF (Kroupa et al. 1993). 


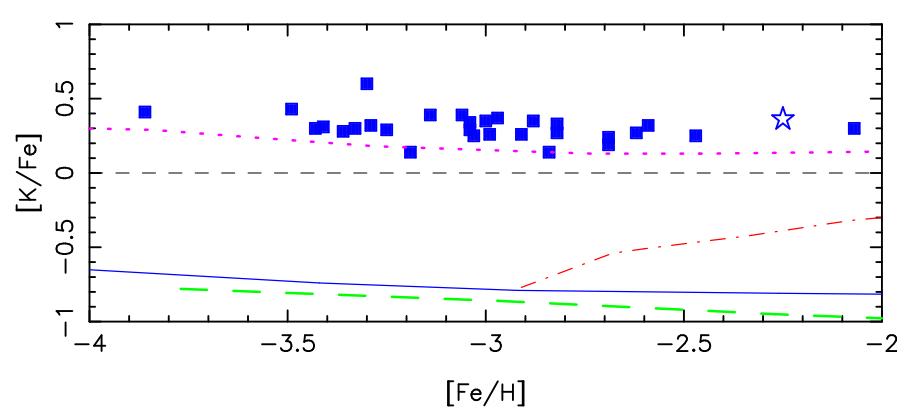

Fig. 9. $[\mathrm{K} / \mathrm{Fe}]$ vs. $[\mathrm{Fe} / \mathrm{H}]$ for metal-poor stars with $[\mathrm{Fe} / \mathrm{H}]<-2.0$ (blue squares), plus our result for HD 84937 (blue star symbol). The theoretical predictions of Timmes et al. (1995; red dot-dashed line), Goswami \& Prantzos (2000; pink dotted line), Kobayashi et al. (2011; blue thick line), and Matteucci (2016; green dashed line) are also represented.

Following Kobayashi et al. (2011) the underproduction of K in the theoretical massive supernovae/hypernovae could be the consequence of a neglect of the $v$ process. When the core of massive stars collapses, a large flux of neutrinos is emitted and these neutrinos interact with heavy elements through neutral-current reactions producing in particular $\mathrm{F}$ and $\mathrm{K}$.

Finally the HST has permitted an extension of the pattern of the light elements produced by the explosion of the progenitor of a very old, metal-poor, unevolved star whose composition is highly representative of the earliest stages of prior massive star nucleosynthesis. These elements are a key to a better understanding of the nucleosynthetic processes that have formed the elements in the early massive supernovae/hypernovae. It is very important that future access to the UV region of the spectra be preserved. This region is a very powerful tool for the study of the early phases of the Galactic evolution.

Acknowledgements. We are grateful to Lyudmila Mashonkina for a very useful and competent report and for the communication of several unpublished nonLTE corrections. We also thank Piercarlo Bonifacio who computed the ATLAS model and WIDTH profiles for us to check the Ci lines. This work was supported by the "Programme National de Physique Stellaire" and the "Programme National de Cosmologie et Galaxies" (CNRS-INSU). Partial support for R. Peterson was provided by NASA under HST GO-14161. R. Peterson thanks her GO-14161 coinvestigators Tom Ayres for his assistance in acquiring and reducing the HST spectra and Robert Kurucz for his advice in analysing them. A. J. Gallagher acknowledges the funding by FONDATION MERAC and the matching fund granted by the Scientific Council of Observatoire de Paris. B. Barbuy was partially supported by FAPESP, CNPq, and CAPES.

\section{References}

Abia, C., \& Mashonkina, L. 2004, MNRAS, 350, 1127

Alvarez, R., \& Plez, B. 1998, A\&A, 330, 1109

Amarsi, A. M., Lind, K., Asplund, M., Barklem, P. S., \& Collet, R. 2016, MNRAS, 463, 1518

Andrievsky, S. M., Spite, M., Korotin, S. A., et al. 2010, A\&A, 509, A88

Anstee, S. D., \& O'Mara, B. J. 1991, MNRAS, 253, 549

Barklem, P. S., \& O'Mara, B. J. 2000, MNRAS, 311, 535

Barklem, P. S., Piskunov, N., \& O'Mara, B. J. 2000, A\&AS, 142, 467

Bergemann, M., Lind, K., Collet, R., Magic, Z., \& Asplund, M. 2012, MNRAS, 427, 27

Bonifacio, P., Spite, M., Cayrel, R., et al. 2009, A\&A, 501, 519
Bonifacio, P., Caffau, E., Spite, M., et al. 2015, A\&A, 579, A28

Caffau, E., \& Ludwig, H.-G. 2007, A\&A, 467, L11

Caffau, E., Bonifacio, P., Faraggiana, R., et al. 2005, A\&A, 441, 533

Caffau, E., Bonifacio, P., Faraggiana, R., \& Steffen, M. 2011a, A\&A, 532, A98

Caffau, E., Ludwig, H.-G., Steffen, M., Freytag, B., \& Bonifacio, P. 2011b, Sol. Phys., 268, 255

Caffau, E., Andrievsky, S., Korotin, S., et al. 2016, A\&A, 585, A16

Carney, B. W., \& Latham, D. W. 1987, AJ, 93, 116

Cescutti, G., Matteucci, F., Caffau, E., \& François, P. 2012, A\&A, 540, A33

de Bruijne, J. H. J., \& Eilers, A.-C. 2012, VizieR Online Data Catalog: III/54

Ducati, J. R. 2002, VizieR Online Data Catalog: II/237

Fabbian, D., Asplund, M., Carlsson, M., \& Kiselman, D. 2006, A\&A, 458, 899

Fabbian, D., Nissen, P. E., Asplund, M., Pettini, M., \& Akerman, C. 2009, A\&A, 500, 1143

François, P. 1986, A\&A, 165, 183

Freytag, B., Steffen, M., Ludwig, H.-G., et al. 2012, J. Comput. Phys., 231, 919

Gallagher, A. J., Steffen, M., Caffau, E., et al. 2016a, [arXiv: 1610.04427]

Gallagher, A. J., Caffau, E., Bonifacio, P., et al. 2016b, A\&A, 593, A48

Gehren, T., Shi, J. R., Zhang, H. W., Zhao, G., \& Korn, A. J. 2006, A\&A, 451, 1065

Goswami, A., \& Prantzos, N. 2000, A\&A, 359, 191

Gustafsson B., Edvardsson B., Eriksson K., et al. 2008, A\&A, 486, 951

Jacobson, H. R., Thanathibodee, T., Frebel, A., et al. 2014, ApJ, 796, L24

Kobayashi, C., Umeda, H., Nomoto, K., Tominaga, N., \& Ohkubo, T. 2006, ApJ, 653,1145

Kobayashi, C., Karakas, A. I., \& Umeda, H. 2011, MNRAS, 414, 3231

Korotin, S. A. 2008, Odessa Astron. Pub., 21, 42

Korotin, S.A. 2009a, Astron. Z., 86, 702

Korotin, S. A. 2009b, Astron. Rep., 53, 651

Kroupa, P., Tout, C. A., \& Gilmore, G. 1993, MNRAS, 262, 545

Lodders, K., Palme, H., \& Gail, H.-P. 2009, Landolt Börnstein, New Series (Berlin: Springer Verlag)

Lai, D. K., Johnson, J. A., Bolte, M., \& Lucatello, S. 2007, ApJ, 667, 1185

Lawler, J. E., Guzman, A., Wood, M. P., Sneden, C., \& Cowan, J. J. 2013, ApJS, 205,11

Lawler, J. E., Sneden, C., \& Cowan, J. J. 2015, ApJS, 220, 13

Lind, K., Melendez, J., Asplund, M., Collet, R., \& Magic, Z. 2013, A\&A, 554, A96

Ludwig, H.-G., Caffau, E., Steffen, M., et al. 2009, Mem. Soc. Astron. It., 80, 711

Mashonkina, L., Gehren, T., Shi, J.-R., Korn, A. J., \& Grupp, F. 2011, A\&A, 528, A 87

Masseron, T., Plez, B., Van Eck, S., et al. 2014, A\&A, 571, A47

Matteucci, F. 2016, J. Phys. Conf. Ser., 703, 012004

Molaro, P., Levshakov, S. A., D’Odorico, S., Bonifacio, P., \& Centurión, M. 2001, ApJ, 549, 90

Moore, C.E. 1945, Contributions from the Princeton University Observatory, 20,1

Peterson, R. C., \& Kurucz, R. L. 2015, ApJS, 216, 1

Peterson, R. C., \& Schrijver, C. J. 1997, ApJ, 480, L47

Placco, V. M., Beers, T. C., Reggiani, H., \& Meléndez, J. 2016, ApJ, 829, L24

Planck Collaboration VII. 2016, A\&A, 594, A7

Plez, B. 2012, http: //2012ascl . soft05004P

Roederer, I. U., Jacobson, H. R., Thanathibodee, T., Frebel, A., \& Toller, E. 2014, ApJ, 797, 69

Roederer, I. U., Placco, V. M., \& Beers, T. C. 2016, ApJ, 824, L19

Shi, J. R., Gehren, T., Mashonkina, L., \& Zhao, G. 2009, A\&A, 503, 533

Sneden, C., Cowan, J. J., Kobayashi, C., et al. 2016, ApJ, 817, 53

Spite, M., Cayrel, R., Plez, B., et al. 2005, A\&A, 430, 655

Spite, M., Caffau, E., Andrievsky, S. M., et al. 2011, A\&A, 528, A9

Spite, M., Andrievsky, S. M., Spite, F., et al. 2012, A\&A, 541, A143

Takeda, Y., \& Takada-Hidai, M. 2011, PASJ, 63, 547

Takeda, Y., Zhao, G., Chen, Y.-Q., Qiu, H.-M., \& Takada-Hidai, M. 2002, PASJ, 54, 275

Takeda, Y., Hashimoto, O., Taguchi, H., et al. 2005, PASJ, 57, 751

Takeda, Y., Kaneko, H., Matsumoto, N., et al. 2009, PASJ, 61, 563

Timmes, F. X., Woosley, S. E., \& Weaver, T. A. 1995, ApJS, 98, 617

VandenBerg, D. A., Bond, H. E., Nelan, E. P., et al. 2014, ApJ, 792, 110

West, C., \& Heger, A. 2013, ApJ, 774, 75

Woosley, S. E., \& Weaver, T. A. 1995, ApJS, 101, 181

Zhao, G., Mashonkina, L., Yan, H. L., et al. 2016, ApJ, 833, 225 